K. KWON

KODAI MATH. J.

19 (1996), 378-387

\title{
NONEXISTENCE OF FINITE ORDER SOLUTIONS OF CERTAIN SECOND ORDER LINEAR DIFFERENTIAL EQUATIONS
}

\author{
KI-Ho KwoN
}

\section{Introduction and statements of results}

Let $\rho(g)$ denote the order of an entire function $g$. Consider the second order linear differential equation

$$
f^{\prime \prime}+A(z) f^{\prime}+B(z) f=0,
$$

where $A(z)$ and $B(z) \not \equiv 0$ are entire functions. It is known that if $\rho(B)<\rho(A)$ $\leqq 1 / 2$, then every solution $f \neq 0$ of $(1)$ is of infinite order [3], [6], [9]. In [7], the author proved the following

THEOREM A. Let $A(z)$ and $B(z)$ be entire functions such that $\rho(A)<\rho(B)$ or $\rho(B)<\rho(A)<1 / 2$. Then every solution $f \not \equiv 0$ of (1) satisfies

$$
\varlimsup_{r \rightarrow \infty} \frac{\log \log T(r, f)}{\log r} \geqq \max \{\rho(A), \rho(B)\},
$$

where $T(r, f)$ is the Nevanlinna characteristic of $f$ (see [5]).

In the case that $\rho(A)>1 / 2$ and $\rho(B)<\rho(A)$, the possibility of solutions $f \not \equiv 0$ of finite order of (1) remains open. In fact, if $\rho(A)$ is a positive integer and $\rho(B)<\rho(A)$, (1) may have nonconstant solutions of finite order [3, Examples 1 and 2$]$.

Here we give a partial result for the case that $\rho(A)$ is not a positive integer with $\rho(A)>1$ and $\rho(B)<\rho(A)$ by proving the following

THEOREM 1. Suppose that $A(z)$ is an entire function of finite nonintegral order with $\rho(A)>1$, and that all the zeros of $A(z)$ lie in the angular sector $\theta_{1} \leqq \arg z \leqq \theta_{2}$ satisfying

$$
\theta_{2}-\theta_{1}<\frac{\pi}{q+1}
$$

The author was supported by the Wharangdae Research Institute, Korea Military Academy, 1995.

Received December 18, 1995 ; revised June 24, 1996. 
if $q$ is odd, and

$$
\theta_{2}-\theta_{1}<\frac{(2 q-1) \pi}{2 q(q+1)}
$$

if $q$ is even, where $q$ is the genus of $A(z)$. Let $B(z)$ be an entire function with $0<\rho(B)<1 / 2$. Then every nonconstant solution $f$ of (1) has infinite order with

$$
\varlimsup_{r \rightarrow \infty} \frac{\log \log T(r, f)}{\log r} \geqq \rho(B) .
$$

Let $P(z)$ and $Q(z)$ be nonconstant polynomials, and let $h_{1}(z)$ and $h_{2}(z) \not \equiv 0$ be entire functions satisfying $\rho\left(h_{1}\right)<\operatorname{deg} P$ and $\rho\left(h_{2}\right)<\operatorname{deg} Q$, where $\operatorname{deg} R$ denotes the degree of a polynomial $R$. Then every nonconstant solution of the second order linear differential equation

$$
f^{\prime \prime}+h_{1}(z) e^{P(z)} f^{\prime}+h_{2}(z) e^{Q(z)} f=0
$$

has infinite order if $\operatorname{deg} P \neq \operatorname{deg} Q$ (p. 419, [3]). If $\operatorname{deg} P=\operatorname{deg} Q$, then (2) may have nonconstant solutions of finite order. Indeed, $f(z)=z$ solves $f^{\prime \prime}+z e^{z} f^{\prime}$ $-e^{z} f=0$.

In this note, we shall investigate the growth of the nonconstant solutions of (2) in the case that $\operatorname{deg} P=\operatorname{deg} Q$.

THEOREM 2. Let $P(z)$ and $Q(z)$ be nonconstant polynomials such that

$$
\begin{aligned}
& P(z)=a_{n} z^{n}+a_{n-1} z^{n-1}+\cdots+a_{1} z+a_{0}, \\
& Q(z)=b_{n} z^{n}+b_{n-1} z^{n-1}+\cdots+b_{1} z+b_{0}
\end{aligned}
$$

for some complex numbers $a_{\imath}, b_{i}(i=0,1,2, \cdots, n)$ with $a_{n} \neq 0, b_{n} \neq 0$ and let $h_{1}(z)$ and $h_{2}(z) \neq \equiv$ be entire functions satisfying $\rho\left(h_{1}\right)<\operatorname{deg} P$ and $\rho\left(h_{2}\right)<\operatorname{deg} Q$. Then the following four statements hold:

(i) If either $\arg a_{n} \neq \arg b_{n}$ or $a_{n}=c b_{n}$ with $0<c<1$, then every nonconstant solution $f$ of (2) has infinite order with

$$
\varlimsup_{r \rightarrow \infty} \frac{\log \log T(r, f)}{\log r} \geqq n .
$$

(ii) Let $a_{n}=b_{n}$ and $\operatorname{deg}(P-Q)=m \geqq 1$, and let the orders of $h_{1}(z)$ and $h_{2}(z)$ be less than $m$. Then every nonconstant solution $f$ of (2) has infinite order with

$$
\varlimsup_{r \rightarrow \infty} \frac{\log \log T(r, f)}{\log r} \geqq m \text {. }
$$

(iii) Let $a_{n}=c b_{n}$ with $c>1$ and $\operatorname{deg}(P-c Q)=m \geqq 1$. Suppose that $\rho\left(h_{1}\right)<m$ and $h_{2}(z)$ is an entire function with $0<\rho\left(h_{2}\right)<1 / 2$. Then every nonconstant solution $f$ of (2) has infinite order with 


$$
\varlimsup_{r \rightarrow \infty} \frac{\log \log T(r, f)}{\log r} \geqq \rho\left(h_{2}\right)
$$

(iv) Let $a_{n}=c b_{n}$ with $c \geqq 1$, and let $P(z)-c Q(z)$ be a constant. Suppose that $\rho\left(h_{1}\right)<\rho\left(h_{2}\right)<1 / 2$. Then every nonconstant solution $f$ of $(2)$ has infinite order with

$$
\varlimsup_{r \rightarrow \infty} \frac{\log \log T(r, f)}{\log r} \geqq \rho\left(h_{2}\right)
$$

\section{Preliminary lemmas}

For the proofs of the theorems we need the following lemmas.

LEMMA B [4]. Let $f(z)$ be a nonconstant entire function, and let $\alpha>1$ and $\varepsilon>0$ be given constants. Then the following two statements hold:

(i) There exist a constant $c>0$ and a set $E_{1} \subset[0, \infty)$ having finte linear measure such that for all $z$ satisfying $|z|=r \notin E_{1}$ we have

$$
\left|\frac{f^{(k)}(z)}{f(z)}\right| \leqq c\left[T(\alpha r, f) r^{\varepsilon} \log T(\alpha r, f)\right]^{k}, \quad k \in N .
$$

(ii) There exist a constant $c>0$ and $a$ set $E_{2} \subset[0,2 \pi)$ having linear measure zero such that if $\phi_{0} \in[0,2 \pi)-E_{2}$, then there is a constant $R_{0}=R_{0}\left(\phi_{0}\right)>0$ so that for all $z$ satisfying $\arg z=\psi_{0}$ and $|z|=r \geqq R_{0}$, we have

$$
\left|\frac{f^{(k)}(z)}{f(z)}\right| \leqq c[T(\alpha r, f) \log T(\alpha r, f)]^{k}, \quad k \in \boldsymbol{N} .
$$

For $E \subset[0, \infty)$, the upper linear density of $E$ is defined by

$$
\overline{\operatorname{dens}} E=\varlimsup_{r \rightarrow \infty} \frac{m(E \cap[0, r])}{r},
$$

where $m(F)$ is the linear measure of a set $F$.

LEMMA C [1]. Let $f(z)$ be an entire function of order $\rho$ where $0<\rho<1 / 2$, and let $\varepsilon>0$ be a given constant. Then there exists a set $E_{3} \subset[0, \infty)$ with $\overline{\operatorname{dens}} E_{3} \geqq 1-2 \rho$ such that for all $z$ satisfying $|z|=r \in E_{3}$, we have

$$
|f(z)| \geqq \exp \left(r^{\rho-\varepsilon}\right) \text {. }
$$

\section{Proof of Theorem 1}

The proof of the theorem depends heavily on the following lemma, which gives information of the minimum moduli of entire functions having their zeros in certain angular sectors. 
LEMMA 3. Let $f(z)$ be an entire function of finite nonintegral order $\rho$ and of genus $q \geqq 1$. Suppose that for any given $\varepsilon>0$, all the zeros of $f(z)$ have their arguments in the following set of real numbers:

if $q$ is odd, and

$$
S(q, \varepsilon)=\left\{\theta:|\theta| \leqq \frac{\pi}{2(q+1)}-\varepsilon\right\}
$$

$$
S(q, \varepsilon)=\left\{\theta: \frac{\pi}{2 q}+\varepsilon \leqq|\theta| \leqq \frac{3 \pi}{2(q+1)}-\varepsilon\right\}
$$

if $q$ is even. Then for any $c>1$, there exists a real number $R>0$ such that

for all $r \geqq R$.

$$
|f(-r)| \leqq \exp \left(-c r^{q}\right)
$$

Proof. Let $z=r e^{i \theta}$ with $r>0,|\theta|<\pi$. Then, from the well-known representation due to Valiron [10], we have

$$
\begin{aligned}
\log E\left(-\frac{z}{r_{n}}, q\right) & =\log \left\{\left(1+\frac{z}{r_{n}}\right) \exp \left(-\frac{z}{r_{n}}+\frac{z^{2}}{2\left(r_{n}\right)^{2}}-\cdots+(-1)^{q} \frac{z^{q}}{q\left(r_{n}\right)^{q}}\right)\right\} \\
& =(-1)^{q} \int_{r_{n}}^{\infty} \frac{z^{q+1}}{t^{q+1}(z+t)} d t
\end{aligned}
$$

for any $r_{n}>0$, where $E(w, q)$ is an elementary factor. Taking real parts, we get

$$
\log \left|E\left(-\frac{z}{r_{n}}, q\right)\right|=(-1)^{q} r^{q+1} \int_{r_{n}}^{\infty} \frac{t \cos (q+1) \theta+r \cos q \theta}{t^{q+1}\left(t^{2}+2 t r \cos \theta+r^{2}\right)} d t .
$$

If $\theta \in S(q, \varepsilon)$, then there is a $\delta>0$ such that

$$
(-1)^{q} \cos (q+1) \theta \leqq-\delta \text { and }(-1)^{q} \cos q \theta \leqq-\delta .
$$

Hence, for $\theta \in S(q, \varepsilon)$,

$$
\log \left|E\left(-\frac{r e^{-i \theta}}{r_{n}}, q\right)\right| \leqq-\delta r^{q+1} \int_{r_{n}}^{\infty} \frac{d t}{t^{q+1}(t+r)} .
$$

By the canonical representation of $f(z)$, we may set

$$
f(z)=z^{m} e^{P(z)} g(z)
$$

for some nonnegative integer $m$, where $P(z)$ is a polynomial with $\operatorname{deg} P \leqq q$ and $g(z)=\Pi E\left(z / a_{n}, q\right)$ with $a_{n}=r_{n} e^{i \theta_{n}}, r_{n}>0$ and $\theta_{n} \in S(q, \varepsilon)$. Since $f(z)$ has nonintegral order $\rho$, we have $\rho(g)=\rho$ and $q<\rho<q+1$. Let $n(t)$ be the number of zeros of $g(z)$ in the disk $|z| \leqq t$; then it follows from (3) that for all $r>0$,

$$
\log |g(-r)|=\Sigma \log \left|E\left(\frac{-r}{a_{n}}, q\right)\right|
$$




$$
\begin{aligned}
& =\Sigma \log \left|E\left(-\frac{r e^{-i \theta} n}{r_{n}}, q\right)\right| \\
& \leqq-\delta r^{q+1} \sum \int_{r_{n}}^{\infty} \frac{d t}{t^{q+1}(t+r)} \\
& =-\delta r^{q+1} \int_{0}^{\infty} \frac{n(t) d t}{t^{q+1}(t+r)} .
\end{aligned}
$$

Now, $\operatorname{deg} P \leqq q$ implies that there are real numbers $c>1$ and $R_{1}>0$ such that

$$
|P(z)| \leqq c r^{q}
$$

for all $|z|=r \geqq R_{1}$. Since $\rho>q$ and

$$
\varlimsup_{r \rightarrow \infty} \frac{\log n(r)}{\log r}=\rho,
$$

there is an $R_{2}>R_{1}$ satisfying

$$
n\left(R_{2}\right) \geqq \frac{12 c q\left(R_{2}\right)^{q}}{\delta} .
$$

Furthermore we may presume that $R_{2}$ is so large that for any $r>2 R_{2}$, we have

$$
\begin{aligned}
\int_{0}^{\infty} \frac{n(t) d t}{t^{q+1}(t+r)} & \geqq \int_{R_{2}}^{r} \frac{n(t) d t}{t^{q+1}(t+r)} \\
& \geqq \frac{n\left(R_{2}\right)}{2 r} \int_{R_{2}}^{r} \frac{d t}{t^{q+1}} \\
& =\frac{n\left(R_{2}\right)}{2 q r}\left(\frac{1}{\left(R_{2}\right)^{q}}-\frac{1}{r^{q}}\right) \\
& \geqq \frac{n\left(R_{2}\right)}{4 q\left(R_{2}\right)^{q} r} .
\end{aligned}
$$

Thus (4), (5) and (6) imply that

$$
\begin{aligned}
\log |g(-r)| & \leqq-\delta r^{q+1} \int_{0}^{\infty} \frac{n(t) d t}{t^{q+1}(t+r)} \\
& \leqq \frac{-\delta n\left(R_{2}\right)}{4 q\left(R_{2}\right)^{q}} r^{q} \leqq-3 c r^{q}
\end{aligned}
$$

for all $r>2 R_{2}$. Therefore

$$
\begin{aligned}
\log |f(-r)| & =m \log r+\operatorname{Re} P(-r)+\log |g(-r)| \\
& \leqq-c r^{q}
\end{aligned}
$$

for all $r>R$ with $R=2 R_{2}$.

Proof of the theorem. Rotating properly the axes of the complex plane, we may assume that all the zeros of $A(z)$ have their arguments in the set 
$S(q, \varepsilon)$ defined in Lemma 3 for some $\varepsilon>0$. Hence, by Lemma 3 , there exists a positive real number $R$ such that for all $r \geqq R$, we have

$$
\min _{|z|=r}|A(z)| \leqq 1 \text {. }
$$

If $f$ is a nonconstant solution of (1), it follows from (1) that

$$
|B(z)| \leqq\left|\frac{f^{\prime \prime}(z)}{f(z)}\right|+|A(z)|\left|\frac{f^{\prime}(z)}{f(z)}\right| .
$$

Hence we deduce from Lemma $C$ and (8) that if $0<\sigma<\rho(B)$, then there is a set $E_{3} \subset[0, \infty)$ with a positive upper density such that for all $z$ satisfying $|z|=r \in E_{3}$, we have

$$
\exp \left(r^{\sigma}\right) \leqq\left|\frac{f^{\prime \prime}(z)}{f(z)}\right|+|A(z)|\left|\frac{f^{\prime}(z)}{f(z)}\right| .
$$

Thus Lemma B and (9) imply that there is a set $E \subset[0, \infty)$ with positive upper density such that for all $z$ satisfying $|z|=r \in E$, we have

$$
\exp \left(r^{\sigma}\right) \leqq(1+|A(z)|) T(2 r, f)^{3} .
$$

Therefore we deduce from (7) and (10) that

$$
\exp \left(r^{\sigma}\right) \leqq 2 T(2 r, f)^{3}
$$

for all $r \in E \cap[R, \infty)$. Thus we conclude that $f(z)$ has infinite order with

$$
\varlimsup_{r \rightarrow \infty} \frac{\log \log T(r, f)}{\log r} \geqq \sigma .
$$

Hence the result of the theorem follows, since $\sigma$ is an arbitrary number less than $\rho(B)$.

\section{Proof of Theorem 2}

In the proof of the theorem, we use the following basic property of polynomials: Let $n$ be a positive integer, and let $P(z)=a_{n} z^{n}+a_{n-1} z^{n-1}+\cdots+a_{1} z+a_{0}$ with $a_{n}=\alpha_{n} e^{i \theta_{n}}, \alpha_{n}>0$. For given $\varepsilon, 0<\varepsilon<\pi / 4 n$, we introduce $2 n$ closed angles

$$
D_{\jmath}:-\frac{\theta_{n}}{n}+(2 j-1) \frac{\pi}{2 n}+\varepsilon \leqq \theta \leqq-\frac{\theta_{n}}{n}+(2 j+1) \frac{\pi}{2 n}-\varepsilon \quad(j=0,1, \cdots, 2 n-1) .
$$

Then, there exists a positive number $R=R(\varepsilon)$ such that

$$
\operatorname{Re} P(z)>\alpha_{n} r^{n}(1-\varepsilon) \sin n \varepsilon
$$

if $|z|=r>R$ and $z \in D_{\jmath}$, where $j$ is even, while

$$
\operatorname{Re} P(z)<-\alpha_{n} r^{n}(1-\varepsilon) \sin n \varepsilon
$$


if $|z|=r>R$ and $z \in D_{\jmath}$, where $j$ is odd (see pp. 253-255, [8]).

Suppose that $h_{1} \equiv 0$. Then if $f$ is a nonconstant solution of (2), it follows from Theorem A that

$$
\varlimsup_{r \rightarrow \infty} \frac{\log \log T(r, f)}{\log r} \geqq n .
$$

Hence all four statements of Theorem 2 are true. Thus, in the following proofs, we always assume that $h_{1} \neq 0$. We also assume that $f$ is a nonconstant solution of (2).

(i) Suppose first that $\arg a_{n} \neq \arg b_{n}$. Then there exist real numbers $\alpha>0$, $R_{1}$ and $\theta_{1}<\theta_{2}$ such that for all $r \geqq R_{1}$ and $\theta_{1} \leqq \theta \leqq \theta_{2}$, we have

$$
\begin{aligned}
& \operatorname{Re} P\left(r e^{i \theta}\right)<0, \\
& \operatorname{Re} Q\left(r e^{i \theta}\right)>\alpha r^{n} .
\end{aligned}
$$

Let $\beta$ satisfy $\rho\left(h_{\imath}\right)<\beta<n(i=1,2)$. Then there is an increasing sequence $\left\{r_{k}\right\}$ of positive real numbers converging to infinity as $k \rightarrow \infty$ such that for $i=1,2$, we have

$$
-\left(r_{k}\right)^{\beta}<\log \left|h_{i}\left(r_{k} e^{i \theta}\right)\right|<\left(r_{k}\right)^{\beta}
$$

for all $k \in \boldsymbol{N}$ and $\theta \in[0,2 \pi$ ) (for the left inequality of (12), see Theorem 3.7.1, $[2])$. We deduce from Lemma $B$ that there exist real numbers $\theta_{0} \in\left(\theta_{1}, \theta_{2}\right)$, $R_{2}>0$ such that for all $z$ satisfying $z=r e^{i \theta_{0}}$ and $r \geqq R_{2}$, we have

$$
\left|\frac{f^{(\jmath)}(z)}{f(z)}\right| \leqq T(2 r, f)^{3}
$$

for $\jmath=1$ and 2 . It follows from (2) that

$$
\left|h_{2}(z) e^{Q(z)}\right| \leqq\left|\frac{f^{\prime \prime}(z)}{f(z)}\right|+\left|h_{1}(z) e^{P(z)}\right|\left|\frac{f^{\prime}(z)}{f(z)}\right| .
$$

Hence, calculating at the points $z_{k}=r_{k} e^{i \theta_{0}}$ with $r_{k} \geqq \max \left\{R_{1}, R_{2}\right\}$, we get from (11), (12), (13) and (14) that

$$
e^{-\left(r_{k}\right)^{\beta}} e^{\alpha\left(r_{k}\right)^{n}} \leqq\left(1+e^{\left(r_{k}\right) \beta}\right) T\left(2 r_{k}, f\right)^{3} .
$$

Thus, $n>\beta$ implies

$$
\varlimsup_{r \rightarrow \infty} \frac{\log \log T(r, f)}{\log r}-\geqq n
$$

Suppose now that $a_{n}=c b_{n}$ with $0<c<1$. Since $\operatorname{deg} Q>\operatorname{deg}(P-c Q)$, there exist real numbers $\alpha>0, \lambda, R_{3}$ and $\theta_{1}<\theta_{2}$ such that

$$
\begin{aligned}
& \operatorname{Re} Q\left(r e^{i \theta}\right)>\alpha r^{n}, \\
& \operatorname{Re}\left\{P\left(r e^{i \theta}\right)-c Q\left(r e^{i \theta}\right)\right\}<\lambda
\end{aligned}
$$


for all $r>R_{1}$ and $\theta \in\left(\theta_{1}, \theta_{2}\right)$. It follows from (2) that

$$
\left|h_{2}(z) e^{(1-c) Q(z)}\right| \leqq\left|e^{-c Q(z)}\right|\left|\frac{f^{\prime \prime}(z)}{f(z)}\right|+\left|h_{1}(z) e^{P(z)-c Q(z)}\right|\left|\frac{f^{\prime}(z)}{f(z)}\right| .
$$

Calculating at the points $z_{k}=r_{k} e^{i \theta_{0}}$ with $r_{k} \geqq \max \left\{R_{2}, R_{3}\right\}$, we deduce from (12), (13), (15) and (16) that

$$
e^{-\left(r_{k}\right)^{\beta}} e^{(1-c) \alpha\left(r_{k}\right)^{n}} \leqq\left(e^{(-c) \alpha\left(r_{k}\right)^{n}}+e^{\left(r_{k}\right)^{\beta}} e^{\lambda}\right) T\left(2 r_{k}, f\right)^{3} .
$$

Thus, $n>\beta$ and $0<c<1$ imply

$$
\varlimsup_{r \rightarrow \infty} \frac{\log \log T(r, f)}{\log r} \geqq n .
$$

(ii) Suppose that $a_{n}=b_{n}$ and $\operatorname{deg}(Q-P)=m, 1 \leqq m<n$. Then there exist real numbers $\alpha>0, R_{1}$ and $\theta_{1}<\theta_{2}$ such that

$$
\begin{aligned}
& \operatorname{Re} P\left(r e^{i \theta}\right) \geqq 0, \\
& \operatorname{Re}\left\{Q\left(r e^{i \theta}\right)-P\left(r e^{i \theta}\right)\right\} \geqq \alpha r^{m}
\end{aligned}
$$

for all $r>R_{1}$ and $\theta \in\left(\theta_{1}, \theta_{2}\right)$. If $\rho\left(h_{\imath}\right)<m(i=1,2)$, then there is an increasing sequence $\left\{r_{k}\right\}$ of real numbers satisfying (12) for some $\beta<m$. It follows from (2) that

$$
\left|h_{2}(z) e^{Q(z)-P(z)}\right| \leqq\left|e^{-P(z)}\right|\left|\frac{f^{\prime \prime}(z)}{f(z)}\right|+\left|h_{1}(z)\right|\left|\frac{f^{\prime}(z)}{f(z)}\right| .
$$

Hence we deduce from (12), (13), (17) and (18) that

$$
e^{-\left(r_{k}\right)^{\beta}} e^{\alpha\left(r_{k}\right)^{m}} \leqq r_{k}\left(1+e^{\left(r_{k}\right)^{\beta}}\right) T\left(2 r_{k}, f\right)^{3}
$$

for all sufficiently large $r_{k}$. Thus, $m>\beta$ implies

$$
\varlimsup_{r \rightarrow \infty} \frac{\log \log T(r, f)}{\log r} \geqq m .
$$

(iii) Suppose that $a_{n}=c b_{n}$ with $c>1$ and $\operatorname{deg}(P-c Q)=m \geqq 1$, and that $\rho\left(h_{1}\right)<m$ and $h_{2}(z)$ is an entire function with $0<\rho\left(h_{2}\right)<1 / 2$. Since $\operatorname{deg}(P-c Q)$ $<\operatorname{deg} Q$, there exist a positive real number $\alpha$ and a continuous curve $C$ tending to infinity such that for all $z \in C$ with $|z|=r$, we have

$$
\begin{aligned}
& \operatorname{Re} Q(z)=0, \\
& \operatorname{Re}\{P(z)-c Q(z)\} \leqq-\alpha r^{m} .
\end{aligned}
$$

Let $\beta$ satisfy $\rho\left(h_{1}\right)<\beta<m$. Then there exists a positive real number $R_{1}$ such that for all $z$ satisfying $|z|=r>R_{1}$, we have

$$
\left|h_{1}(z)\right| \leqq \exp \left(r^{\beta}\right) .
$$


Furthermore, if $0<\gamma<\rho\left(h_{2}\right)$, then by Lemma $C$, there exists a set $E_{3} \subset[0, \infty)$ with positive upper density such that for all $z$ satisfying $|z|=r \in E_{3}$, we have

$$
\left|h_{2}(z)\right| \geqq \exp \left(r^{r}\right) \text {. }
$$

It follows from (2) that

$$
\left|h_{2}(z) e^{(1-c) Q(z)}\right| \leqq\left|e^{-c Q(z)}\right|\left|\frac{f^{\prime \prime}(z)}{f(z)}\right|+\left|h_{1}(z) e^{P(z)-c Q(z)}\right|\left|\frac{f^{\prime}(z)}{f(z)}\right| .
$$

Calculating at $z=r e^{i \theta}$ on $C$, we deduce from (19), (20), (21), (22) and Lemma B that

$$
e^{r^{r}} \leqq r\left(1+e^{r^{\beta}} e^{-\alpha r^{m}}\right) T(2 r, f)^{3}
$$

for some unbounded set of $r$. Thus, $m>\beta$ implies

$$
\varlimsup_{r \rightarrow \infty} \frac{\log \log T(r, f)}{\log r} \geqq \gamma .
$$

Therefore

$$
\varlimsup_{r \rightarrow \infty} \frac{\log \log T(r, f)}{\log r} \geqq \rho\left(h_{2}\right),
$$

since $\gamma$ is an arbitrary real number less than $\rho\left(h_{2}\right)$.

(iv) Let $a_{n}=c b_{n}$ with $c \geqq 1$ and let $P(z)-c Q(z)$ be a constant. Suppose that $\rho\left(h_{1}\right)<\rho\left(h_{2}\right)<1 / 2$. If $\alpha$ and $\beta$ satisfy $\rho\left(h_{1}\right)<\beta<\alpha<\rho\left(h_{2}\right)$, then Lemma C and the definition of the order imply that there exists a set $E \subset[0, \infty)$ with a positive upper density such that for all $z$ satisfying $|z|=r \in E$, we have

$$
\begin{gathered}
\left|h_{1}(z)\right| \leqq \exp \left(r^{\beta}\right), \\
\left|h_{2}(z)\right| \geqq \exp \left(r^{\alpha}\right) .
\end{gathered}
$$

Let $C$ be a continuous curve tending to infinity on which $\operatorname{Re} Q(z)=0$. Then we deduce from (21), (22), (23) and Lemma B that

$$
e^{r^{\alpha}} \leqq r\left(1+b e^{r^{\beta}}\right) T(2 r, f)^{3}
$$

for some real number $b$, and for some unbounded set of $r$. Hence $\alpha>\beta$ implies

$$
\varlimsup_{r \rightarrow \infty} \frac{\log \log T(r, f)}{\log r} \geqq \alpha .
$$

Thus

$$
\varlimsup_{r \rightarrow \infty} \frac{\log \log T(r, f)}{\log r} \geqq \rho\left(h_{2}\right),
$$

since $\alpha$ is an arbitrary real number less than $\rho\left(h_{2}\right)$. 


\section{REFERENCES}

[1] P.D. BARRY, Some theorems related to the $\cos \pi \rho$ theorem, Proc. London Math. Soc. (3), 21 (1970), 334-360.

[2] R.P. BoAs, Entire Functions, Academic Press, New York, 1954.

[3] G. GundERsEn, Finite order solutions of second order linear differential equations, Trans. Amer. Math. Soc., 305 (1988), 415-429.

[4] G. Gundersen, Estimates for the logarithmic derivative of a meromorphic function, plus similar estimates, J. London Math. Soc. (2), 37 (1988), 88-104.

[5] W. Hayman, Meromorphic Functions, Clarendon Press, Oxford, 1964.

[6] S. Hellerstein, J. Miles and J. Rossi, On the growth of solutions of $f^{\prime \prime}+g f^{\prime}$ $+h f=0$, Trans. Amer. Math. Soc., 323 (1991), 693-706.

[7] K. Kwon, On the growth of entire functions satisfying second order linear differential equations, Bull. Korean Math. Soc. (2), 33 (1996), 487-496.

[8] A.I. Markushevich, Theory of Functions of a Complex Variable (Vol. II), Prentice-Hall, New Jersey, 1965.

[9] M. OzAw A, On a solution of $w^{\prime \prime}+e^{-z} w^{\prime}+(a z+b) w=0$, Kodai Math. J., 3 (1980), 295-309.

[10] G. Valiron, Sur les fonctions entières d'ordre fini et d'ordre nul, et en particulier les fonctions à correspondance régulière, Ann. Fac. Sci. Univ. Toulouse, 3 (1913), 117-257.

Department of Mathematics

Korea Military Academy

SEOUL, KOREA 